\title{
Boswell, John, Jack Corbett, und R. A. W. Rhodes (2019): The Art and Craft of Comparison
}

Cambridge: Cambridge University Press. 174 Seiten. $£ 22.99$

\author{
Norma Osterberg-Kaufmann
}

Online publiziert: 16 . November 2020

(C) Der/die Autor(en) 2020

Das Buch ,The Art and Craft of Comparison ist in der Reihe „Strategies for Social Inquiry“ bei Cambridge University Press erschienen. Es verortet sich selbst in der Universalismus-Partikularismus-Debatte, also der Frage nach der einen universellen Wahrheit oder der vielen Wahrheiten in Abhängigkeit spezifischer historischer und sozialer Kontexte. Außerdem sieht das Buch sich als alternativen Ansatz einer vergleichenden Politikwissenschaft jenseits der Spaltung in eine naturalistische Sozialwissenschaft (,natural science“) einerseits und geisteswissenschaftliche Area Studies (,humanistic art“) andererseits. John Boswell, Jack Corbett und R. A. W. Rhodes möchten mit ihrem Ansatz eine Brücke über diese Kluft zwischen quasi-naturwissenschaftliche und kulturelle Betrachtungsweise politischer Phänomene bilden. Die Monografie sieht sich eher als Ratgeber denn als Lehrbuch und verfolgt zwei Ziele: Erstens möchten die Autoren KollegInnen in der Anfangsphase ihrer Karriere mit der vergleichenden interpretativen Forschung inspirieren. Zweitens möchte sie all jenen, die bereits in einer interpretativen Tradition arbeiten, eine Argumentationshilfe und einen Referenzrahmen bieten. Neben der Vermittlung von grundsätzlichem Handwerkszeug will der Band auch Standards zur Erhebung von Daten sowie zur Beurteilung interpretativer vergleichender Forschung setzen. Das Buch soll zudem eine zum Nachdenken anregende und zugängliche Flankierung des etablierten Kanons der qualitativen und humanistischen Sozialwissenschaften sein. Auch wenn laut der Autoren quantitative Daten Teil der interpretativen vergleichenden Methode sein können, so liegt der Fokus des vorliegenden Buches dennoch auf der qualitativen Forschung, wobei die Autoren versuchen, diese ihrer Meinung nach artifizielle Unterscheidung nicht aufzugreifen.

N. Osterberg-Kaufmann ( $\triangle)$

Institut für Sozialwissenschaften, Humboldt-Universität zu Berlin, Berlin, Deutschland

E-Mail: norma.osterberg-kaufmann@hu-berlin.de 
Boswell, Corbett und Rhodes skizzieren eine Reihe von Prinzipien, Standards und Ansätzen, die zentral für die interpretative vergleichende Forschung sind. Ihre Argumentation in den Kapiteln 2 und 3, in denen die theoretischen Grundlagen des interpretativen Ansatzes vorgestellt werden, lässt sich folgendermaßen zusammenfassen: Im Unterschied zu einer eher zu Quantifizierung neigenden Vergleichenden Politikwissenschaft, in deren Fokus Gesetze, Regeln, Korrelationen zwischen sozialen Kategorien oder deduktive Modelle stehen, konzentriert sich die interpretative Forschung auf Bedeutungen, Überzeugungen und Diskurse. Der interpretativen vergleichenden Methode geht es um ein ganzheitliches Verständnis davon, wie und warum AkteurInnen handeln. Bestimmt ist dieses Handeln von einem Netz von Überzeugungen (,web of meaning“, S. 21), Diskursen und Traditionen, in denen sich die AkteurInnen befinden und die ihre Glaubenssätze (,,beliefs“, S. 21) beeinflussen. Die interpretative vergleichende Forschung kontrastiert den naturalistischen Fokus auf Verallgemeinerbarkeit, isolierende Variablen, kausale Schlussfolgerungen und Widerlegung mit dem interpretatorischen Schwerpunkt auf ,thick description“, „web of meaning“, komplexer Spezifizierung und intersubjektiver Objektivität. Ein zentraler Grundsatz der interpretativen Ausrichtung ist, dass menschliches Handeln historisch bedingt ist. Hier bestehen Anknüpfungspunkte zur Travelling-Prämisse, die konzentriert auf Konzepte davon ausgeht, dass diese sich über Raum und Zeit wandeln. InterpretationsforscherInnen fragen in der Konsequenz dieses Grundsatzes nicht, ob die Ergebnisse generalisierbar sind, sondern ob sie im Kontext funktionieren. Gleichzeitig gehen sie davon aus, dass Vergleiche dennoch Sinn ergeben und sich insbesondere der interpretative Ansatz in besonderem Maße eignet, ungewöhnliche Vergleiche, wie der des französischen Präsidenten mit einem oder einer DorfvorsteherIn im ländlichen Indien, anzustellen.

Als die drei zentralen Grundbegriffe der Analyse in der interpretativen Forschung werden den LeserInnen nachfolgend Glaubenssätze (,beliefs“, S. 21), Traditionen und Dilemmata vorgestellt. Glaubenssätze helfen den AkteurInnen bei der Interpretation ihrer Umwelt. Glaubenssätze oder, allgemeiner formuliert, Überzeugungen entstehen nicht in einem Vakuum, sondern sind Teil eines gemeinsamen gesellschaftlichen Erbes und einer gemeinsamen Konstruktion. Hier wird die konstruktivistische Perspektive, die dem gesamten Ansatz als solchem zugrunde liegt, im Buch aber nicht einmal als entsprechende Referenz verwendet wird, deutlich.

Das Konzept der Tradition, als zweiter Grundbegriff, erklärt, warum Menschen handeln, wie sie handeln. Menschen verstehen ihre Erfahrungen anhand von Theorien, die sie sozusagen geerbt haben. Dieses soziale Erbe ist ein notwendiger, sich ständig weiterentwickelnder Rahmen für die Überzeugungen, die Menschen annehmen, und für die Handlungen, die sie ausführen. Eine Tradition ist also eine Reihe von Erkenntnissen, die jemand während einer andauernden, manchmal sogar episodischen Sozialisation erhält.

Das Dilemma schließlich, als dritter Kernbegriff des interpretativen Vergleichs, entsteht für ein Individuum oder eine Institution, wenn eine neue Idee im Gegensatz zu bestehenden Überzeugungen und der damit verbundenen Tradition steht. Wann immer jemand eine neue Überzeugung oder Handlung annimmt, muss er oder sie seine bestehenden Überzeugungen und Praktiken anpassen, um Platz für den Neuankömmling zu schaffen. Diskussionswürdig wäre hier jedoch, ob jede neue Idee 
zwangsläufig zu einem Dilemma für die AkteurInnen führen muss, sprich in einem Spannungsverhältnis mit den gegebenen Überzeugungen und Traditionen steht oder ob sie nicht auch harmonieren können.

Der Vergleich wird schließlich möglich, indem untersucht wird, wie AkteurInnen in unterschiedlichen Kontexten bemerkenswert ähnliche Dilemmata artikulieren. Die Ähnlichkeiten zwischen Dilemmata erlauben es interpretierenden ForscherInnen, plausible Vermutungen zu entwickeln, die über den unmittelbaren Kontext, in dem sie ursprünglich entwickelt wurden, hinaus nachhallen können.

In den Kapiteln 4, 5, 6 und 7 entwickeln Boswell, Corbett und Rhodes Faustregeln zum Forschungsdesign, zur Feldstudie als Methode der Datenerhebung, zur Datenanalyse und zum Schreibprozess, anhand deren sich AnwenderInnen der interpretativen vergleichenden Methode im Forschungsprozess orientieren können. Dabei stechen die vielzähligen konkreten Beispiele aus der Feldforschung sowie Erfahrungen der Autoren in wissenschaftlichen Auseinandersetzungen positiv hervor. Auch die lebendige Sprache, in der das Buch geschrieben ist, macht es in besonderem Maße zugänglich. Spannend, überraschend und ungewohnt, in der Logik des vorgestellten Forschungsansatzes jedoch absolut kohärent, ist das Schlusskapitel in Form einer Retrospektive. Hier reflektieren die Autoren ihre eigene Rolle bei dessen Entstehen, die jeweilige Motivation beim Schreiben, die Auswahl der Zielgruppe und fassen schließlich vor diesem Hintergrund die zentralen Argumente und den zentralen Beitrag des Bandes zusammen.

Empfehlenswert ist dieses Buch nicht nur für jene, die bereits interpretativ vergleichend arbeiten oder mit dem Gedanken spielen, sondern für alle vergleichenden PolitikwissenschaftlerInnen. Es regt an, über den Tellerrand des Forschungsmainstreams zu blicken, sich in einer konstruktivistischen Denkweise zu versuchen, inklusive deren methodischen Konsequenzen, sowie möglicherweise Inspiration zu finden, wie jenseits eines starren Universalismus oder Relativismus/Partikularismus eine immer diverser werdende Welt vergleichend zu erforschen sein könnte.

Funding Open Access funding enabled and organized by Projekt DEAL.

Open Access Dieser Artikel wird unter der Creative Commons Namensnennung 4.0 International Lizenz veröffentlicht, welche die Nutzung, Vervielfältigung, Bearbeitung, Verbreitung und Wiedergabe in jeglichem Medium und Format erlaubt, sofern Sie den/die ursprünglichen Autor(en) und die Quelle ordnungsgemäß nennen, einen Link zur Creative Commons Lizenz beifügen und angeben, ob Änderungen vorgenommen wurden.

Die in diesem Artikel enthaltenen Bilder und sonstiges Drittmaterial unterliegen ebenfalls der genannten Creative Commons Lizenz, sofern sich aus der Abbildungslegende nichts anderes ergibt. Sofern das betreffende Material nicht unter der genannten Creative Commons Lizenz steht und die betreffende Handlung nicht nach gesetzlichen Vorschriften erlaubt ist, ist für die oben aufgeführten Weiterverwendungen des Materials die Einwilligung des jeweiligen Rechteinhabers einzuholen.

Weitere Details zur Lizenz entnehmen Sie bitte der Lizenzinformation auf http://creativecommons.org/ licenses/by/4.0/deed.de. 